\title{
Barbara Welker
}

\section{Das Archiv der Stiftung Neue Synagoge Berlin - Centrum Judaicum}

Unter ganz anderen Verhältnissen als heute überschrieb Jacob Jacobson, der letzte Leiter des Gesamtarchivs der deutschen Juden, im Juni 1938 einen Artikel im Jüdischen Gemeindeblatt für Berlin mit der Forderung „Schützt Euer Archivgut!“, in dem er die jüdischen Gemeinden aufforderte, vor allem ihre familiengeschichtlich relevanten Dokumente dem Gesamtarchiv in Berlin zukommen zu lassen. ${ }^{1}$ Dennoch ist die Aufgabe, sich des gefährdeten Archivgutes anzunehmen, auch heute noch aktuell.

Vor über 15 Jahren kehrte ein großer Teil der Bestände aus dem früheren Gesamtarchiv der deutschen Juden nach einer langen Odyssee in die Räume in der Oranienburger Straße zurück.Die wechselhafte Geschichte des Archivs soll hier nur kurz dargestellt werden. ${ }^{2}$

Überlegungen zur Einrichtung eines wissenschaftlichen jüdischen Archivs gab es bereits im letzten Drittel des 19. Jahrhunderts. Mehrfach gab es Ansätze, vor allem die mittelalterlichen Quellen und Urkunden zur Geschichte der Juden zu erfassen, zusammenzutragen und auszuwerten. So hatte u.a. der Deutsch-Israelitische Gemeindebund auf Betreiben des Historikers Harry Bresslau 1885 eine Historische Kommission für die Geschichte der Juden in Deutschland gegründet, die allerdings nur wenige Jahre Bestand hatte. ${ }^{3}$

1 Jüdisches Gemeindeblatt für Berlin, Nr. 26, 26.6.1938. S. 3; vorher bereits in der CV-Zeitung, Nr. 22, 2.6.1938. Das Gesamtarchiv musste sich zu diesem Zeitpunkt bereits Gesamtarchiv der Juden in Deutschland nennen.

2 Zur Geschichte und Tätigkeit des Gesamtarchivs siehe auch: Welker, Barbara: Das Gesamtarchiv der deutschen Juden. In: „Tuet auf die Pforten“. Die Neue Synagoge 1866-1995. Begleitbuch zur ständigen Ausstellung der Stiftung „Neue Synagoge Berlin - Centrum Judaicum“. Hrsg. von Hermann Simon u. Jochen Boberg. Berlin 1995. S. 227-234; Welker, Barbara: Das Gesamtarchiv der deutschen Juden - Zentralisierungsbemühungen in einem föderalen Staat. In: Jüdisches Archivwesen. Beiträge zum Kolloquium aus Anlass des 100. Jahrestages der Gründung des Gesamtarchivs der deutschen Juden, zugleich 10. Archivwissenschaftliches Kolloquium der Archivschule Marburg, 13.-15. Sept. 2005. Hrsg. von Frank M. Bischoff u. Peter Honigmann. Marburg 2007 (Veröffentlichungen der Archivschule Marburg, Institut für Archivwissenschaft, 45). S. 39-73. 3 Die Kommission löste sich aufgrund innerer Kontroversen - unter anderem um die Einbeziehung des Historikers Heinrich Graetz - nach wenigen Jahren wieder auf. Sie gab 1887-1892 die erste Zeitschrift für die Geschichte der Juden sowie mehrere wichtige Quellenpublikationen zur Geschichte der Juden in Deutschland heraus. 
Der schlesische Historiker und Archivar Ezechiel Zivier ${ }^{4}$ machte schließlich 1903 in einer Sitzung der Lessing-Loge Breslau des Unabhängigen Ordens B'nai B'rith (UOBB) den Vorschlag zur Errichtung eines „allgemeinen Archivs für die Juden Deutschlands“, um „eine Zentrale zu schaffen, wohin jede Gemeinde, eine jede jüdische Körperschaft ihre älteren Akten und Dokumente, die für die laufende Geschäftsführung nicht mehr von Belang sind, zur weiteren Aufbewahrung und Nutzbarmachung für geschichtliche und andere Forschungen abgeben könnte"5.

Diese Idee wurde von der Großloge für Deutschland des UOBB und dem Deutsch-Israelitischen Gemeindebund aufgegriffen. Das Gesamtarchiv sollte im Wesentlichen zwei Aufgaben erfüllen: einerseits die Sammlung, Sicherung, Erschließung und Auswertung der historischen Akten der jüdischen Gemeinden, Organisationen und Vereine in Deutschland an einer zentralen Stelle, andererseits die Erfassung und Dokumentation von Quellen zur Geschichte der Juden in staatlichen und anderen Archiven, um eine umfassende Erforschung der Geschichte der Juden in Deutschland zu ermöglichen. ${ }^{6}$

Schließlich konnte am 1. Oktober 1905 das Gesamtarchiv eröffnet werden, zunächst in gemieteten Räumen in der Lützowstraße 15 in Berlin-Tiergarten ${ }^{7}$; 1910 zog das Archiv in das neu errichtete Verwaltungsgebäude der Jüdischen Gemeinde zu Berlin in der Oranienburger Str. 28/29 - in diesem Gebäude befand sich auch die Bibliothek der Gemeinde. ${ }^{8}$

4 Zu Ezechiel Zivier (1868-1925) s. den Nachruf von Ismar Elbogen in: Mitteilungen des Gesamtarchivs der deutschen Juden (im Folgenden: MittGA) 6 (1926). S. 112-113. Eine Dissertation über Zivier legte die polnische Historikerin Barbara Kalinowska-Wójcik 2005 an der Schlesischen Universität Katowice vor, s. auch ihren Beitrag: Kalinowska-Wójcik, Barbara: Ezechiel Zivier - życie i działalność archiwisty i historyka na Górnym Śląsku na przełomie XIX i XX wieku. In: Szkice archiwalno-historiczne, 4 (2008). S. 35-47.

5 Zivier, Ezechiel: Eine archivalische Informationsreise. In: Monatsschrift für Geschichte und Wissenschaft des Judentums (im Folgenden: MGWJ), 49 (1905). S. 209-254, S. 209.

6 Zum Programm des Archivs siehe Täubler, Eugen: Zur Einführung. In: MittGA, 1 (1908/09), 1. S. $1-8$.

7 Mitteilung über das Gesamtarchiv der deutschen Juden. In: MGWJ, 50 (1906). S. 246-247, S. 246.

8 Der Einzug erfolgte im März 1910, allerdings fand erst Ende Dezember die feierliche Eröffnung statt, s. Bericht über die Tätigkeit des Gesamtarchivs der deutschen Juden. In: MittGA, 3 (1911/12). S. 55-84, v.a. S. 55-75. 
Erster Leiter des Archivs war der Historiker Eugen Täubler (1879-1953) ${ }^{9}$, auf ihn folgte 1920 Jacob Jacobson (1888-1968) ${ }^{10}$.

Da es für die Archivalien jüdischer Gemeinden und Organisationen keine festgelegte Zuständigkeit gibt, beruhte die Abgabe der Akten durch die Gemeinden auf Freiwilligkeit. Täubler und andere Vertreter des Archivs schrieben alle Gemeinden im Deutschen Reich an und unternahmen mehrfach Reisen zu den Gemeinden, um sie zur Abgabe ihrer alten Akten zu bewegen.

In der Regel wurden die Gemeindeakten auf der Grundlage von Depositalverträgen übergeben.

Bis Dezember 1907 hatten 167 jüdische Gemeinden ihre Akten abgeliefert ${ }^{11}$, 1908 waren es Akten aus 178 Gemeinden sowie Akten des Deutsch-Israelitischen Gemeindebundes und Spenden von Privatpersonen ${ }^{12}$. Beim Umzug in die Oranienburger Straße 1910 hatten 273 Gemeinden Akten abgeliefert ${ }^{13}$, bis zum Jahr 1926 waren es 344 Gemeinden. ${ }^{14}$ Bei den abgegebenen Gemeindebeständen überwogen Akten kleinerer Gemeinden - die größeren jüdischen Gemeinden unter-

9 Bericht über die Tätigkeit (wie Anm. 8). Eugen Täubler (1879 Gostyn/Posen-1953 Cincinnati) hatte an der Berliner Universität sowie am Rabbinerseminar und an der Lehranstalt (später Hochschule) für die Wissenschaft des Judentums studiert. Eine Ausbildung als Archivar erhielt er am Preußischen Geheimen Staatsarchiv in Berlin-Dahlem. Zur Biographie Täublers s. SternTäubler, Selma: Einleitung. Eugen Täubler und die Wissenschaft des Judentums. In: Täubler, Eugen: Aufsätze zur Problematik jüdischer Geschichtsschreibung 1908-1950. Hrsg. u. eingeleitet von Selma Stern-Täubler. Tübingen 1977 (Schriftenreihe wissenschaftlicher Abhandlungen des Leo Baeck Instituts 36). S. VII-XXIV; Scharbaum, Heike: Zwischen zwei Welten. Wissenschaft und Lebenswelt am Beispiel des deutsch-jüdischen Historikers Eugen Täubler (1879-1953). Münster 2001.

10 Jacob Jacobson (1888 Schrimm/Posen-1968 Bad Neuenahr), Sohn eines Rabbiners, studierte Klassische Philologie, Geschichte und Germanistik und promovierte 1919 in Marburg. Bis zu seiner Deportation 1943 blieb sein Name mit der weiteren Geschichte des Gesamtarchivs verbunden. Zu seiner Biographie s. die Nachrufe von Jersch-Wenzel, Stefi: Zum Tode Jacob Jacobsons. In: Jahrbuch für die Geschichte Mittel- und Ostdeutschlands 18 (1969). S. 698-704, und von Brilling, Bernhard, in: Der Archivar 22 (1969). Sp. 234-236, sowie vor allem zum Zeitraum 1933-1945 Jersch-Wenzel, Stefi u. Jersch, Thomas: Jacob Jacobson - deutscher Jude und Archivar (1888-1968). In: Archive und Gedächtnis. Festschrift für Botho Brachmann. Hrsg. von Friedrich Beck, Eckart Henning, Joachim-Felix Leonhard, Susanne Paulukat u. Olaf B. Rader. Potsdam 2005 (Potsdamer Studien 18) S. 547-585.

11 Mitteilung des Gesamtarchivs der deutschen Juden. In: Mitteilungen vom Deutsch-Israelitischen Gemeindebund, 69 (Dez. 1907). S. 24-25.

12 Geschäftsbericht. In: MittGA, 1 (1908/09). S. 45-46.

13 Bericht über die Tätigkeit (wie Anm. 8). S. 81-83.

14 Geschäftsbericht. In: MittGA, 6 (1926). S. 116-121. Das ist nur ein kleiner Teil aller Gemeinden - vor dem Ersten Weltkrieg gab es über 2.000, Anfang der 1930er Jahre noch rund 1.500 jüdische Gemeinden (mit Filialgemeinden) in Deutschland. 
hielten oft eigene Archive (z.B. Breslau, Hamburg, Frankfurt/Main, München, Königsberg). ${ }^{15}$

Zwischen 1908 und 1926 wurden sechs Jahrgänge der Mitteilungen des Gesamtarchivs der deutschen Juden veröffentlicht, die neben Tätigkeitsberichten und Akteninventaren einzelner Gemeinden auch Spezialinventare (Hinweise auf Akten zur Geschichte der Juden in staatlichen Archiven) und wissenschaftliche Beiträge zur Geschichte der Juden in Deutschland enthielten.

Unter der Leitung von Jacob Jacobson erfolgte bereits seit den 1920er Jahren eine verstärkte Orientierung auf familiengeschichtliche Quellen und Forschungen. ${ }^{16}$ Nach 1933 wurde das Gesamtarchiv in stark wachsendem Umfang Anlaufstelle für familiengeschichtliche Anfragen und sammelte daher verstärkt Geburts-, Heirats- und Sterberegister, Beschneidungsbücher, Memorbücher u.ä. Einerseits musste das Archiv vor allem nach dem Erlass der „Nürnberger Gesetze“ 1935 zunehmend Auskünfte für so genannte Abstammungsnachweise erteilen andererseits nahm nach 1933 auch innerhalb der jüdischen Gemeinschaft gerade unter den Bedingungen des wachsenden Drucks von außen das Interesse an der Erforschung der eigenen Familiengeschichte, aber auch der jüdischen Lokal- und Regionalgeschichte zu. Wiederholt wies Jacobson mit Anzeigen und Artikeln in jüdischen Zeitungen auf die Bedeutung der Bestände des Gesamtarchivs für familiengeschichtliche Nachforschungen hin und bat um die Ablieferung weiterer Quellen. ${ }^{17}$

Ab 1938/39 hatte das Gesamtarachiv faktisch seine Selbstständigkeit verloren, nach dem Novemberpogrom 1938 wurden die Bestände des Gesamtarchivs beschlagnahmt und das Archiv vorübergehend geschlossen. Jacob Jacobson durfte das Archiv erst nach einigen Wochen wieder betreten. ${ }^{18}$

15 Lediglich die Berliner Gemeinde hatte 1910 ihr Archiv dem Gesamtarchiv angeschlossen, s. Bericht über die Tätigkeit (wie Anm. 8). S. 61. Vgl. auch Welker: Gesamtarchiv (wie Anm. 2). S. 63-68.

16 So waren sowohl Jacob Jacobson als Privatperson als auch das Gesamtarchiv als Institution Mitglied in der 1924 gegründeten Gessellschaft für jüdische Familienforschung, s. Mitgliederliste. In: Jüdische Familienforschung. Mitteilungen der Gesellschaft für jüdische Familienforschung, 1 (1925), 2. S. 43-46, S. 43.

17 So beispielsweise in einem kurzen Hinweis auf die Öffnungszeiten des Archivs in: Jüdische Rundschau, Nr. 40, 19.5.1933. S. 208. In der vermutlich letzten Veröffentlichung über das Gesamtarchiv schreibt Jacob Jacobson noch über „Beschneidungsbücher als personengeschichtliches Quellenmaterial“. In: Jüdisches Nachrichtenblatt, Nr. 15, 10.4.1942.

18 Jacobson, Jacob Bruchstücke 1939-1945, Leo Baeck Institute New York, Memoirensammlung, M.E. 560. Bl. 2 (teilweise abgedruckt in: Richarz, Monika (Hrsg.): Jüdisches Leben in Deutschland. Bd. 3: Selbstzeugnisse zur Sozialgeschichte 1918-1945. Stuttgart 1982. S. 401-412. Zum Schicksal der Sammlungen im Gebäudekomplex Oranienburger Straße 28-31 (Jüdisches Museum, Gesamtarchiv und Bibliothek der Jüdischen Gemeinde) nach dem Novemberpogrom s. auch: Simon, 
Die Reichsstelle für Sippenforschung richtete in der Oranienburger Straße ihre „Zentralstelle für jüdische Personenstandsregister“ ein und nutzte hierfür auch die Bestände des Gesamtarchivs, Jacob Jacobson musste dem Reichssippenamt zuarbeiten. ${ }^{19}$ Seine geplante Emigration nach England wurde von der Gestapo verhindert, lediglich seine Frau und sein Sohn konnten auswandern. Jacob Jacobson wurde am 19. Mai 1943 nach Theresienstadt deportiert. ${ }^{20}$

Die für genealogische Nachforschungen relevanten Dokumente (Geburts-, Heirats- und Sterberegister, Memorbücher usw.) wurden zusammen mit anderen vom Reichssippenamt gesammelten jüdischen Personenstandsregistern aus den Beständen des Gesamtarchivs herausgenommen, während des Krieges nach Thüringen ausgelagert und dort im Auftrag des Amtes verfilmt. Die Originale sind heute verschollen - vermutlich wurden sie bei Kriegsende vernichtet. ${ }^{21}$

Die historischen Aktenbestände des Gesamtarchivs wurden 1943 an das Preußische Geheime Staatsarchiv in Berlin-Dahlem abgegeben, von wo sie später ausgelagert wurden und nach 1945 zunächst nach Merseburg (Sachsen-Anhalt) gelangten. 1950 wurden die verbliebenen Archivalien der Jüdischen Gemeinde zu Berlin übergeben. ${ }^{22}$

Hermann: Auf der Suche nach einer verlorenen Sammlung. Was geschah nach dem 10. November 1938 mit den Beständen des Berliner Jüdischen Museums? In: Auf der Suche nach einer verlorenen Sammlung. Das Berliner Jüdische Museum (1933-1938). Berlin 2011. S. 17-46, v.a S. 21-23. 19 Das Reichssippenamt war eine dem Reichsministerium des Innern nachgeordnete Behörde; aus der ursprünglichen Dienststelle beim Sachverständigen für Rasseforschung beim Reichsministerium des Innern entstand 1935 die Reichsstelle für Sippenforschung, ab 1940 das Reichssippenamt. Das Amt sollte genealogische Quellen erfassen und sichern und diente als maßgebliche Stelle für Auskünfte und Gutachten über die „arische Abstammung“ in Zweifelsfällen. Zu Geschichte und Aufgaben des Amtes s. Schulle, Diana Das Reichssippenamt. Eine Institution nationalsozialistischer Rassenpolitik. Berlin 2001. Zum „Verhältnis“ zwischen Gesamtarchiv und Reichssippenamt ab 1938 siehe Jersch-Wenzel u. Jersch: Jacob Jacobson (wie Anm. 10). S. 570-575.

20 Jersch-Wenzel u. Jersch: Jacob Jacobson (wie Anm. 10). S. 569, Anm. 113. Nach der Befreiung des Ghettos Theresienstadt 1945 reiste er direkt nach England zu seiner Familie, ebd. S. 578-580. 21 Die von der Firma Gatermann gefertigten Mikrofilme blieben erhalten - zu ihrer Geschichte, vor allem für Hessen, s. Heinemann, Hartmut: Das Schicksal der jüdischen Personenstandsregister. Die verschlungenen Wege der Gatermann-Filme. In: Bischoff u. Honigmann (Hrsg.): Archivwesen (wie Anm. 2). S. 193-215.

22 Brachmann-Teubner, Elisabeth: Sources for the History of the Jews from the Eighteenth Century to the Twentieth Century in the Archives of the former DDR. In: Leo Baeck Institute Year Book (im Folgenden: LBIYB), 38 (1993). S. 391-408, S. 406. Der Kantor der Israelitischen Religionsgemeinde Leipzig, Werner Sander, fertigte zuvor ein Verzeichnis der erhaltenen Bestände an, das in den Beständen des Verbandes der Jüdischen Gemeinden in der DDR überliefert ist (CJA, 5B1, Nr. 10). 
Etwa die Hälfte der erhaltenen Akten gelangte in den 1950er Jahren in die Central Archives for the History of the Jewish People in Jerusalem (vor allem ältere Archivalien und Hebraica) ${ }^{23}$; die in Berlin verbliebenen Akten wurden 1958 als Depositum an das Deutsche Zentralarchiv in Potsdam (später Zerntrales Staatsarchiv der DDR) übergeben, wo sie seit den 1980er Jahren erschlossen wurden. ${ }^{24}$ Diese Bestände kamen 1990 in die Obhut des Bundesarchivs und wurden - wie eingangs geschrieben - 1996 an das Centrum Judaicum übergeben, kehrten also nach über 50 Jahren in die alten Räume des Gesamtarchivs zurück. ${ }^{25}$

\section{Bestände}

Das Archiv der Stiftung Neue Synagoge Berlin - Centrum Judaicum verwahrt heute insgesamt über 500 lfm Archiv- und Sammlungsgut, rund 2.500 Mikrofilme und über 10.300 Mikrofiches.

Aus den Beständen des früheren Gesamtarchivs befinden sich heute rund $230 \mathrm{lfm}$ Archivmaterialien aus dem Zeitraum 17. Jahrhundert bis $1945 \mathrm{im}$ Archiv, wobei der Schwerpunkt der Überlieferung im Zeitraum 19. und Anfang des 20. Jahrhunderts liegt. ${ }^{26}$

Die Gruppe der Gemeindebestände ist die bei weitem umfangreichste, insgesamt handelt es sich um rund 10.200 Akten aus 400 jüdischen Gemeinden, die meisten aus dem früheren Deutschen Reich.

23 Rein, Denise: Die Bestände der ehemaligen jüdischen Gemeinden Deutschlands in den Central Archives for the History of the Jewish People in Jerusalem. Ein Überblick über das Schicksal der verschiedenen Gemeindearchive: in: Der Archivar, 55 (2002). S. 318-327, S. 320-322.

24 Brachmann-Teubner: Sources (wie Anm. 22). S. 406.

25 Weitere Teilbestände aus dem früheren Gesamtarchiv der deutschen Juden befinden sich heute im Archiv des Leo Baeck Institute (jetzt Center for Jewish History) in New York, im so genannten Sonderarchiv in Moskau sowie im Jüdisch-Historischen Institut in Warschau. Bei den in Moskau liegenden Materialien handelt es sich vor allem um eine Zeitungsausschnittsammlung (Fonds 1194, Sammlung Neumann), vgl. die Übersicht auf der privaten Homepage www.sonderarchiv.de (18.9.2014). Gemeint ist der Berliner Arzt und langjährige Stadtverordnete Salomon Neumann (1819 Pyritz - 1908 Berlin), zu ihm s. jetzt Günter Regneri, Salomon Neumann. Sozialmediziner - Statistiker - Stadtverordneter, (Jüdische Miniaturen, Bd. 107), Berlin 2011.

26 Die Findbücher für alle hier vorhandenen Bestände sind nahezu vollständig abgedruckt in den beiden Bänden Quellen zur Geschichte der Juden in den Archiven der neuen Bundesländer. Hrsg. von Stefi Jersch-Wenzel u. Reinhard Rürup. Bd. 6: Stiftung „Neue Synagoge Berlin - Centrum Judaicum“. München 2001. Ein kleiner Teil der Akten ist wegen seines sehr schlechten Erhaltungszustandes nicht verzeichnet und nicht mehr benutzbar. 
Der mit Abstand größte Gemeindebestand stammt mit rund 1.200 Akten aus Berlin (1827-1945) - trotzdem ist die Überlieferung der Jüdischen Gemeinde zu Berlin bis 1945 nur sehr lückenhaft.

Wichtige Akten, etwa vom Vorstand und den Repräsentanten, sind nicht überliefert; eine dichtere Aktenlage gibt es nur für Teilbereiche der Gemeindetätigkeit, etwa für einzelne Fürsorgeeinrichtungen (Altersheime, Sammelvormundschaft), für Schulen und die Lehrerbildungsanstalt sowie Bau- und Grundstücksakten aus der NS-Zeit.

Die übrigen Gemeindebestände stammen überwiegend aus Preußen (v.a. aus den Provinzen Posen, Schlesien, Hessen-Nassau, Brandenburg), daneben gibt es Akten kleinerer Gemeinden in Bayern, Hessen und Mecklenburg. Südwestdeutsche Gemeinden sind kaum vertreten.

Umfangreichere Gemeindebestände sind auch z.B. aus Allenstein, Beuthen, Borek, Breslau, Bürgel, Darmstadt (mit Landjudenschaft), Dettensee, Elbing, Erfurt, Frankfurt/Oder, Graetz, Halberstadt, Koschmin, Märkisch-Friedland, Offenbach, Pasewalk, Pleschen, Pyritz, Ratibor, Schwedt, Schwersenz, Stargard, Stettin, Strelitz und Sulzbach überliefert.

Die Überlieferungsdichte ist sehr unterschiedlich: während einzelne Gemeindebestände ein breites Spektrum der Gemeindeverwaltung widerspiegeln, sind aus anderen Gemeinden nur die an die zuständige Regierung gesandten Etats und Jahresrechnungen überliefert oder ist nur Sammlungsgut vorhanden (meist Drucksachen wie Statuten und Tätigkeitsberichte von Vereinen und Wohlfahrtseinrichtungen, die vom Gesamtarchiv in einer gesonderten Abteilung gesammelt wurden). ${ }^{27}$

So gut wie nicht überliefert sind die von den Familienforschern in der Regel angefragten Mitgliederverzeichnisse, Steuerlisten, Zu- und Abzugslisten, Personenstandsregister (Geburts-, Heirats- und Sterberegister), Beschneidungsbücher, Memorbücher usw. - die, wie oben beschrieben, zusammen mit anderen Beständen im Reichssippenamt vermutlich als verlorengegangen gelten müssen. ${ }^{28}$

Insgesamt ist die Überlieferung der meisten Gemeinden nur fragmentarisch, häufig liegt ein weiterer Teilbestand in den Central Archives for the History of the Jewish People in Jerusalem. $^{29}$

27 Bericht über die Tätigkeit (wie Anm. 8). S. 76-77.

28 Eine Ausnahme bilden lediglich die für die Jüdische Gemeinde Breslau überlieferten Heiratsregister aus den Jahren 1817-1847 und Sterberegister für die Jahre 1822-1937 sowie einzelne Register für jüdische Gemeinden in Polen, deren Zugang ungeklärt ist.

29 Vgl. Rein: Bestände (wie Anm. 23). Die Findbücher für die Bestände der deutschen jüdischen Gemeinden sind online recherchierbar. 
Eine zweite, sehr heterogene Bestandsgruppe innerhalb der Gesamtarchivbestände umfasst die meist nur kleinen Bestände von Rabbinaten und Gemeindeverbänden der Länder bzw. preußischen Provinzen. Interessant und umfangreicher sind in dieser Gruppe u.a. die Bestände 1, 75 B Fu 1 (Provinzial-Vorsteheramt der Israeliten in Fulda) mit 105 Akten aus den Jahren 1766-1923, der Bestand 1, 75 B Ka 1 (Oberrat der Israeliten Badens), 87 Akten aus den Jahren 1813-1940, und der Bestand 1, 75 B Schw 2 (Distriktsrabbinat Schweinfurt/Niederwerrn), 85 Akten aus den Jahren 1783-1911.

Eine dritte Bestandsgruppe enthält Akten jüdischer Organisationen, darunter vom Deutsch-Israelitischen Gemeindebund (Bestand 1, 75 C Ge 1, 2512 Akten aus dem Zeitraum 1871-1940), dem Verband der deutschen Juden (1, 75 C Ve 1, 449 Akten, 1903-1922), dem Allgemeinen Rabbinerverband in Deutschland (1, 75 C Ra 1, 36 Akten, 1884-1938) sowie der Gesellschaft zur Fördenung der Wissenschaft des Judentums (1, 75 C Ge 4, 69 Akten, 1902-1936).

Leider nicht überliefert sind in unserem Archiv die - häufig angefragten Akten des Centralvereins jüdischer Staatsbürger jüdischen Glaubens und des Hilfsvereins der deutschen Juden. ${ }^{30}$

Die vierte Bestandsgruppe innerhalb des Gesamtarchivs umfasst 18 meist kleinere Nachlässe, darunter ein Teilnachlass von Leo Baeck aus den Jahren 1889-1942 und der schmale, aber bereits sehr intensiv ausgewertete Nachlass der ersten Rabbinerin in Deutschland, Regina Jonas (1902-1944 in Auschwitz ermordet). ${ }^{31}$

Die letzte Bestandsgruppe schließlich enthält Sammlungsgut wie einzelne Dokumente, Druckschriften, Familienpapiere, Zeitungen, Manuskripte usw., deren Provenienz unbekannt oder unerheblich war und die keinem bestehenden Bestand angegliedert werden konnten.

Weitere Bestände gelangten direkt von der Jüdischen Gemeinde zu Berlin oder anderen Gemeinden und Verbänden an unser Archiv.

Aus der Zeit bis 1945 verfügen wir u.a. über die Austrittskartei der Jüdischen Gemeinde zu Berlin ab dem Ende des 19. Jahrhunderts bis 1941/42 - eine wichtige

30 Die jüngeren Akten des Centralvereins - vor allem aus den 1920er und 1930er Jahren - wurden vermutlich 1938 bei der Auflösung des Vereins beschlagnahmt und tauchten 1991 im Moskauer „Sonderarchiv“ auf, s. Barkai, Avraham: The C.V. and its Archives. In: LBIYB, 45 (2000). S. 173-182. Die Akten des Hilfsvereins müssen wohl als verschollen gelten.

31 Die Stiftung Neue Synagoge Berlin - Centrum Judaicum zeigte anlässlich des 75. Jahrestages ihrer Ordination 1935 eine kleine Ausstellung über Regina Jonas (2010/11). Ausgewertet wurde der Nachlass auch von Elisa Klapheck (Fräulein Rabbiner Jonas. Kann die Frau das rabbinische Amt bekleiden. Teetz 2000), die die halachische Arbeit von Regina Jonas aus dem Jahr 1930 edierte und kommentierte. 
Quelle auch deshalb, weil die eigentliche Mitgliederkartei der Gemeinde nicht überliefert ist.

Über das Jahr 1945 hinaus gehen die Registerbände des Jüdischen Krankenhauses, die unserem Archiv in den Jahren 1998 und 2001 übergeben wurden. Es handelt sich vor allem um Aufnahmebücher, daneben um vereinzelte Geburtenbücher (1920-1957) und Sterbebücher (1946-1966). Die Aufnahmebücher für die Patienten bzw. die dazu angefertigten alphabetischen Register umfassen etwa den Zeitraum 1935-1960 (nicht lückenlos). Oft angefragt, aber leider nicht überliefert sind Personalakten der am Krankenhaus tätigen Ärzte.

In Kopie (Mikrofiches) verfügen wir außerdem über die Beisetzungsregister der jüdischen Friedhöfe in der Schönhauser Allee (nur ein kleiner Teil ab etwa 1876) und in Berlin-Weißensee (1880-1990). Die Originale dieser Register befinden sich bei der Friedhofsverwaltung der Jüdischen Gemeinde zu Berlin in Weißensee.

Ein weiterer Bestand sind die Akten der Jüdischen Gemeinde Halle/Saale aus den Jahren 1859-1995. Ein Schwerpunkt dieses Bestandes liegt in der Zeit des Nationalsozialismus (bis 1943), für einzelne Gemeindemitglieder sind akribisch die einzelnen Schritte der Entrechtung und Verfolgung, vom Berufsverbot über die Ablieferung von Radio, Schreibmaschine, Woll- und Pelzsachen, den Verlust der Wohnung und Wohnungseinrichtung und der zwangsweise Umzug in ein Altersheim oder Judenhaus bis hin zur Deportation dokumentiert. Daneben umfasst der Bestand auch eine Reihe von Dokumenten aus der Zeit nach 1945, u.a. zu Wiedergutmachungsangelegenheiten.

Eine interessante Quelle zur Verfolgung der Berliner Juden sind außerdem 352 hier vorhandene Personalakten jüdischer Zwangsarbeiter bei der Berliner Farbenfabrik Warnecke \& Böhm in Berlin-Weißensee aus den Jahren 1939-1943. ${ }^{32}$

Daneben verfügt das Archiv Centrum Judaicum über einige umfangreiche Bestände aus der Zeit nach 1945.

Aus der unmittelbaren Nachkriegszeit stammt eine Kartei jüdischer Antragsteller auf eine Anerkennung als „Opfer des Faschismus“ [bzw. „Opfer der Nürnberger Gesetzgebung"] aus den Jahren 1945 bis etwa 1948 (OdF-Kartei) - für gut $10 \%$ der Antragsteller ist außerdem eine Personalakte überliefert, die meist

32 Die Akten wurden in den Jahren 1991/92 zunächst an ihrem Fundort kopiert. In den Jahren 2003/04 wurde an verschiedenen Stationen in Berlin eine vom Museumsverbund Pankow erarbeitete Ausstellung über die Zwangsarbeit bei der Firma Warnecke \& Böhm gezeigt (s. hierzu den Band: Zwangsarbeit und „Arisierung“. Warnecke \& Böhm - ein Beispiel. Hrsg. vom Bezirksamt Pankow von Berlin in Zusammenarbeit mit der Stiftung Neue Synagoge Berlin - Centrum Judaicum. Berlin 2004). Im Anschluss daran kamen im September 2004 die Originalakten in unser Archiv. 
einen Antrag und einen kurzen Lebenslauf mit der Schilderung der erlittenen Verfolgungsmaßnahmen enthält (rund 3.300 Akten).

Die Jüdische Gemeinde zu Berlin übergab uns einen großen Teil ihrer Akten aus der Zeit nach 1945 - insgesamt rund 220 lfm Archivmaterial. In den Jahren 2000-2002 wurde der Bestand bis zum Jahr 1990 im Rahmen eines von der VWStiftung finanzierten Projekts erschlossen. Die Akten der Ost- und Westberliner Gemeinde wurden in unserem Archiv zusammengefasst, wobei drei Teilbestände gebildet wurden: die Überlieferung der Nachkriegsgemeinde in den Jahren 19451953, Jüdische Gemeinde von Berlin-Ost 1953-1990 und Jüdische Gemeinde zu Berlin-West 1953-1990. Hierzu muss allerdings bemerkt werden, dass die Überlieferungsdichte sehr unterschiedlich ist, von 1953 bis zur Mitte der 1970er Jahre gibt es bereits große Überlieferungslücken, vor allem für den Westteil der Stadt.

Ein weiterer wichtiger Bestand sind die Akten des Verbandes der Jüdischen Gemeinden in der DDR, 1945-1990, der im September 1990 - vor dem Beitritt seiner Mitgliedsgemeinden zum Zentralrat der Juden in Deutschland - beschloss, seine Akten an unser Archiv abzugeben.

Daneben verwalten wir natürlich auch die Registratur des Centrum Judaicum selbst (etwa die Recherche- und Korrespondenzakten zu den hier gezeigten Ausstellungen) sowie die Überlieferung verschiedener Forschungsprojekte, darunter die Projektunterlagen und Korrespondenzakten für das beim ehemaligen Zentralinstitut für Sozialwissenschaftliche Forschung an der Freien Universität Berlin erarbeitete und 1995 veröffentlichte Gedenkbuch Berlins der jüdischen Opfer des Nationalsozialismus.

Eine wichtige Bestandsgruppe sind die Nachlässe, die zu unseren am häufigsten und intensivsten genutzten und ausgewerteten Beständen gehören. (Dies gilt übrigens auch für die Nachlässe innerhalb der Gesamtarchivbestände.) Darunter befindet sich etwa ein Nachlass-Splitter von Gertrud Kolmar (1894-1943), der u.a. das Manuskript des Gedichtzyklus „Das Wort der Stummen“ aus dem Jahr 1933 enthält.

Weitere interessante Nachlässe sind etwa der Teilnachlass des BernsteinUnternehmers Moritz Becker (1830-1901) und seines Sohnes Arthur Becker, Rittergutsbesitzer und sozialdemokratischer Reichstagsabgeordneter (1862-1933) ${ }^{33}$ sowie zahlreiche Dokumente aus dem Nachlass von Hans Rosenthal (1925-1987), die in den vergangenen Jahren mehrfach ausgestellt wurden; daneben die Nach-

33 Ein weiterer Teil dieses Nachlasses befindet sich im Landesarchiv Mecklenburg-Vorpommern, Archiv Greifswald. 
lässe des Rabbiners und Autors Emil Bernhard Cohn (1881-1948) und seiner Schwester Lotte Cohn (1893-1983), einer frühen Architektin in Palästina ${ }^{34}$.

Veröffentlicht wurde auch eine Sammlung von Feldpostbriefen ehemaliger Zöglinge und Mitarbeiter des Berliner Reichenheimschen Waisenhauses aus dem Ersten Weltkrieg, die beim ehemaligen Direktor Dr. Sigmund Feist (1865-1943) erhalten geblieben sind und 1995 dem Centrum Judaicum übergeben wurden. ${ }^{35}$

Andere Nachlässe stammen nicht von prominenten Personen, enthalten aber wichtige Dokumente etwa zur Emigration nach Schanghai, dem Schicksal der Emigrantengemeinschaft in Schanghai und (in diesen Fällen) der Rückkehr nach Deutschland 1947 (Nachlass Denny und Bertha Gottlieb sowie Nachlass Siegfried Sachs).

Die letzte Bestandsgruppe schließlich bilden die Sammlungen. Sie entstanden durch Schenkungen und Kauf von Privatpersonen oder Institutionen und sollen die bestehenden Archivbestände ergänzen.

Bei dem Sammlungsgut handelt es sich überwiegend um Dokumente von Personen und Familien, deren Umfang zu gering ist, um einen Nachlassbestand zu bilden (Familienpapiere wie z.B. Bürgerbriefe, Geburts-, Heirats- und Sterbeurkunden, Ahnentafeln, Familienchroniken und -stammbücher, Traueralben, Briefwechsel, Feldpostbriefe, Kennkarten, Arbeitsbücher, Zeugnisse und Urkunden, Manuskripte, Erinnerungsberichte usw.), daneben Akten von Gemeinden, Institutionen, Vereinen und Firmen sowie Druckschriften, Zeitschriften, Plakate, Flugblätter und Fotos bzw. Fotoalben.

Darüber hinaus gibt es im Centrum Judaicum ein gesondertes Fotoarchiv, das sowohl historische Fotos (z.B. aus dem früheren Berliner Jüdischen Museum) als auch die Dokumentation des Wiederaufbaus der Neuen Synagoge Berlin sowie im Zusammenhang mit Veranstaltungen und Ausstellungen des Hauses entstandene Bilder enthält.

34 Siehe die Biographie: Sonder, Ines: Baumeisterin des Landes Israel. Berlin 2010. Im Jahr 2009 zeigte das Centrum Judaicum eine Ausstellung zum Werk von Lotte Cohn.

35 Feldpostbriefe jüdischer Soldaten 1914-1918. Bearb., kommentiert u. eingeleitet von Sabine Hank u. Hermann Simon. 2 Bde. Teetz 2002. 


\section{Bestandsergänzung, Erschließung und Bestandserhaltung}

Die Sammlungen des Archivs werden kontinuierlich erweitert. In seinen Sammelschwerpunkten lehnt sich das Archiv dabei an das frühere Gesamtarchiv der deutschen Juden an.

Der Schwerpunkt der Überlieferung liegt auf dem Zeitraum bis 1945, für Berlin und die neuen Bundesländer sammeln wir ergänzend $\mathrm{zu}$ den bestehenden Beständen auch Material aus der Zeit nach 1945. Zugänge gelangen meist als Schenkung durch Privatpersonen (Nachlässe, Familienpapiere usw.), in geringem Umfang auch durch Ankauf (Fotos, Postkarten, Broschüren) in unser Archiv, daneben übernehmen wir regelmäßig neuere Akten der Jüdischen Gemeinde zu Berlin. Diese werden fortlaufend registriert; Nachlässe und Bestände jüdischer Gemeinden und Institutionen werden als Bestand erschlossen, Einzeldokumente und kleinere Zugänge den Sammlungen angegliedert. Die Verzeichnung der Bestände erfolgt mit Hilfe des Archivprogramms AUGIAS.

Die aus dem früheren Gesamtarchiv stammenden Bestände wurden bereits vor der Übernahme im Frühjahr 1996 gegen Schimmelbefall behandelt, anschließend teilweise entsäuert und als konservatorische Maßnahme in archivgerechte Mappen und Kartons verpackt. Die Akten selbst, die in Folge der wechselvollen Geschichte der Bestände teilweise starke Schäden aufweisen, wurden - soweit der Erhaltungszustand es zuließ - mikroverfilmt. Einzelne inhaltlich wichtige stark geschädigte Akten konnten in den zurückliegenden Jahren, häufig mit Hilfe von Drittmitteln, etwa des Hauptstadtkulturfonds des Bundes oder durch Stiftungen, restauriert werden.

Daneben wurden alle benutzbaren Akten noch einmal intensiv verzeichnet. Dabei wurde auch die innere Ordnung der Bestände überarbeitet und stärker an die Gliederung im früheren Gesamtarchiv der deutschen Juden angelehnt: Organisation und allgemeine Verwaltung der Gemeinde (Rechtsstellung, Behördenverfügungen, Statuten, Vorstand und Repräsentanten, Mitglieder, Allgemeines), Personalangelegenheiten, Grundstücke und Bauten, Finanzen und Steuern, Kultusangelegenheiten, Wohlfahrt und Unterstützungen, Vereine. Dabei half, dass häufig die alten Signaturen noch auf den Aktendeckeln stehen. Die alten Karteien und Zugangsakten des Gesamtarchivs sind nicht erhalten.

In der 2001 veröffentlichten Beständeübersicht (s. Anm. 26) sind auch die Findbücher des Bestandes Verband der jüdischen Gemeinden in der DDR sowie der bis zum Jahr 2000 bereits übernommenen und erschlossenen Nachlässe abgedruckt. 
Auf der Website der Stiftung Neue Synagoge Centrum Judaicum kann man sich über die vorhandenen Bestände informieren. In den kommenden Jahren sollen sukzessive auch Findbücher online gestellt werden.

Wünschenswert wäre es auch, könnten eines Tages die verstreut überlieferten Bestände der jüdischen Gemeinden und Organisationen in Form virtueller Findbücher im Netz zusammengeführt werden, um die Überlieferungsdichte und auch die Lücken deutlich zu machen und Nutzern die Recherche nach den Quellen zu vereinfachen. ${ }^{36}$

Die Beisetzungsregister im Archiv des jüdischen Friedhofs in Berlin-Weißensee wurden Anfang der 1990er Jahre in Zusammenarbeit mit dem Landesarchiv Berlin mit Mitteln der Stiftung Deutsche Klassenlotterie restauriert und mikroverfilmt. Die Mikrofiches können in unserem Archiv eingesehen werden.

Auch die Quellen aus der unmittelbaren Nachkriegszeit sind wegen der schlechten Papierqualität in dieser Zeit bereits stark in ihrer Erhaltung gefährdet. Die beiden Nachkriegsbestände des Verbandes der Jüdischen Gemeinden in der $D D R$ - dessen Akten immerhin vor über zehn Jahren sicherungsverfilmt wurden und auf Mikrofiche vorliegen - und der Jüdischen Gemeinde zu Berlin zählen zu den am häufigsten nachgefragten und benutzten Beständen.

Gegenwärtig realisieren wir ein Modellprojekt zur Restaurierung von Akten aus der unmittelbaren Nachkriegszeit 1945-1952, um die Erhaltung dieser Akten in den nächsten Jahren planen zu können. ${ }^{37}$

\section{Benutzung des Archivs und Auswertung der Bestände}

Die Benutzung des Archivs ist durch eine schriftliche Anfrage oder persönliche Benutzung möglich. Für Benutzung und Recherchen werden Gebühren erhoben (Ausnahmen gelten etwa für amtliche Anfragen und wissenschaftliche oder heimatgeschichtliche Forschung). Das Archiv steht jedem zur Verfügung, der ein berechtigtes persönliches oder wissenschaftliches Interesse geltend machen

36 Für den sehr zerstreut überlieferten Bestand der ehemaligen jüdischen Gemeinde in Bromberg ist dies vor einigen Jahren geschehen: Quellen zu Geschichte der Juden in polnischen Archiven. Im Auftrag der Berlin-Brandenburgischen Akademie der Wissenschaften hrsg. von Stefi Jersch-Wenzel. Bd. 1: Ehemalige preußische Provinzen Pommern, Westpreußen, Ostpreußen, Preußen, Posen, Grenzmark Posen-Westpreußen, Süd- und Neuostpreußen. München 2003. S. 499-528.

37 Dieses Projekt wird finanziert über die Koordinierungsstelle für die Erhaltung des schriftlichen Kulturgutes (KEK) bei der Staatsbibliothek zu Berlin (Stiftung Preußischer Kulturbesitz). 
kann. Empfehlenswert ist es, sich zunächst in unseren gedruckten Findbüchern und im Internet kundig zu machen und anschließend eine schriftliche Anfrage zu stellen. Persönliche Benutzer kommen in der Regel nach einer vorherigen schriftlichen Anfrage bzw. Recherche in den Findbüchern nach Absprache in unser Archiv.

Für einzelne Bestände können aus Gründen des Personendatenschutzes Benutzungsbeschränkungen bestehen, v.a. für jüngere und personenbezogene Akten oder für Nachlässe und persönliche Dokumente entsprechend der Vereinbarung mit den Nachlassgebern bzw. Spendern.

Im Jahr 2011 wurden über 1.300 überwiegend schriftliche Anfragen bearbeitet - diese Zahl ist seit einigen Jahren konstant mit leicht steigender Tendenz. Während die Zahl amtlicher Anfragen (v.a. Bundesämter für zentrale Dienste und offene Vermögensfragen) zurückging, nahm die Zahl privater und heimatgeschichtlicher Anfragen (z.B. biographische Recherchen im Zusammenhang mit dem Projekt „Stolpersteine“) zu.

Etwa ein Drittel der Anfragen sind familiengeschichtlicher oder privater Natur, danach folgen wissenschaftliche Anfragen und Anfragen von Behörden (Nachweis der Religionszugehörigkeit bzw. der Verfolgung in Rückerstattungsfragen, Ermittlung Angehöriger in Nachlassangelegenheiten, Nachweis der deutschen Staatsangehörigkeit u.ä.). Nur einen kleinen Teil machen Anfragen publizistischer Natur und Anfragen von Rechtsanwälten und kommerziellen Erbenermittlern aus.

Natürlich bilden die Archivbestände auch eine wichtige Grundlage für die Arbeit des Centrum Judaicum selbst. Neben den bereits genannten Quelleneditionen und Ausstellungen werden die Bestände immer wieder für Recherchen und zur Präsentation in den Ausstellungen des Hauses herangezogen - wie für die wichtige Ausstellung Juden in Berlin 1938-1945, die im Jahr 2000 gezeigt wurde, ${ }^{38}$ oder die Ausstellung Zwischen Bleiben und Gehen (2008) mit Biographien überlebender Juden in der Sowjetischen Besatzungszone und der frühen DDR. ${ }^{39}$

38 Begleitband Juden in Berlin 1938-1945. Hrsg. von Beate Meyer u. Hermann Simon. Berlin 2000; engl. Ausgabe: Jews in Nazi Berlin. From Kristallnacht to Liberation. Hrsg. von Beate Meyer, Hermann Simon u. Chana Schütz. Chicago 2009.

39 Begleitband Zwischen Bleiben und Gehen. Juden in Ostdeutschland 1945 bis 1956. Zehn Biographien. Hrsg. von Andreas Weigelt u. Hermann Simon. Berlin 2008. 